\title{
24. STANDARDIZATION OF THE CALCULATION OF NEARLY PARABOLIC COMETARY ORBITS
}

\author{
L. E. NIKONOVA \\ Kazan University, Kazan, U.S.S.R. \\ and \\ N. A. BOKHAN \\ Institute for Theoretical Astronomy, Leningrad, U.S.S.R.
}

\begin{abstract}
A complex of standard programmes has been developed for computing nearly parabolic orbits. The true anomaly is related to the time using Herrick's formulae. Perturbations are calculated by Encke's method.
\end{abstract}

A library of standard programmes (SP) has been compiled in the department of minor planets and comets of the Institute for Theoretical Astronomy. These programmes, written for electronic computers of the type M-20, are intended for serving many of the problems frequently encountered in astronomical calculations, and they are continuously being supplemented.

The standardization of calculations on electronic computers is a new stage in the development of computational techniques, and it enables us to formulate and solve new topical problems in cometary astronomy. Among these problems is the systematic calculation of orbits for all nonperiodic comets, and it has been our purpose to provide a complex of standard programmes for computing definitive, nearly parabolic orbits.

This complex of SP's may be divided into three groups. One group is used for computing perturbations by the major planets, another is for comparison with observations, and the third enables one to carry out a multiple orbit improvement.

The SP's for calculating perturbations are based upon a set of coordinates, stored on magnetic tape, of the eight major planets Venus to Pluto over a period of 400 years (Kazimirchak-Polonskaya, 1967). For calculating the perturbations Encke's method is used, our programme consisting of fourteen SP's written by different authors.

A standard programme by Nikonova causes the coordinates of the major planets during the period of the comet's visibility to be read from the magnetic tape into the computer's high-speed memory. This is used in conjunction with two SP's by Bokhan, which

(1) identify the zone number on the tape and determine the corresponding initial date for the coordinates of the major planets on the magnetic drum and in highspeed memory;

(2) read out the coordinates of the planets from the magnetic drum forward and backward according to the sign of the integration step. The coordinates are interpolated for intervening dates by Lagrange's formula. 
To determine the rectangular heliocentric coordinates of the comet the true anomaly is first calculated using Herrick's (1960) formula:

$$
\begin{aligned}
\frac{k(1+e)^{2}(t-T)}{2 p^{3 / 2}}= & \frac{\sigma}{1+\lambda \sigma^{2}}+\frac{\sigma^{3}}{1+e} \\
& \times\left[\frac{1}{1+\lambda \sigma^{2}}-\frac{1}{3}+\sum_{n=1}^{\infty} \frac{(-1)^{n+1}\left(\lambda \sigma^{2}\right)^{n}}{2 n+3}\right],
\end{aligned}
$$

where

$$
\sigma=\tan \frac{v}{2}, \quad \lambda=\frac{1-e}{1+e},
$$

$k$ being the Gaussian constant, $v$ the true anomaly, $e$ the eccentricity, $T$ the time of perihelion passage, $p$ the parameter, and $t$ a given moment. The unperturbed rectangular coordinates of the comet are obtained from the usual formulae.

The total disturbing acceleration on the comet is calculated by means of an SP by Kazimirchak-Polonskaya, and SP's are then used for integrating the perturbations on the comet in rectangular coordinates. The initial integration table is constructed for seven starting moments, using an SP for obtaining the first and second differences. The orbit is then integrated in groups of three consecutive steps forward (or backward), and the final perturbed rectangular coordinates of the comet are obtained for the total visibility period (Nikonova).

For comparison of the observed and calculated spherical coordinates of the comet the observations are first processed using the programme by N. A. Belyaev, which reduces the coordinates to a standard equinox and calculates the topocentric coordinates of the Sun. The disturbed rectangular coordinates of the comet are interpolated for each observation (the time being corrected for aberration, with the distance from the Earth calculated by successive approximations). The comet's calculated right ascension and declination are obtained from the rectangular coordinates of the comet and the Sun, and then the $(\mathrm{O}-\mathrm{C})$ residuals are printed out and punched onto cards (Bokhan).

The programme for the orbit improvement combines SP's for

(1) the calculation of auxiliary values;

(2) formation of the Eckert-Brouwer differential coefficients using the formulae modified by Dubyago (1949) for $e \simeq 1$ (Nikonova);

(3) formulation of the equations of condition (S. G. Makover, Bokhan);

(4) application of the method of least squares (Makover).

The programme allows the simultaneous treatment of up to 190 observations or normal places. The improved elements, the mean square errors of the unknowns, the mean square error of unit weight and the final residuals are then printed out and punched. The programme has been applied to comet $1963 \mathrm{~V}$ (Nikonova, 1968).

\section{References}

Dubyago, A. D.: 1949, Opredelenie Orbit, Moscow, p. 293 
Herrick, C. E.: 1960, Details of Computational Methods: Space Trajectories, p. 135.

Kazimirchak-Polonskaya, E. I.: 1967, Trudy Inst. Teor. Astron. 12, 24.

Nikonova, L. E.: 1968, Trudy Astron. Obs. Kazan No. 35, 169.

\section{Discussion}

G. Sitarski: What principle do you use for the rejection of observations?

L. E. Nikonova: The observations are rejected by the $3 \sigma$ principle.

M. Bielicki: Did you take into account the weights of the observations in solving the normal equations?

L. E. Nikonova: No. 